\title{
Expression of vascular endothelial growth factor in the growth plate is stimulated by estradiol and increases during pubertal development
}

\author{
Joyce Emons, Andrei S Chagin', Torun Malmlöf1, Magnus Lekman', Åsa Tivesten², \\ Claes Ohlsson' ${ }^{2}$, Jan M Wit, Marcel Karperien ${ }^{\mathbf{3}, \mathbf{4}}$ and Lars Sävendahl ${ }^{\mathbf{1}}$ \\ Department of Paediatrics, Leiden University Medical Center, 2300 ZA Leiden, The Netherlands \\ ${ }^{1}$ Department of Women's and Children's Health, Karolinska Institutet, SE-171 76 Stockholm, Sweden \\ ${ }^{2}$ Division of Endocrinology, Department of Internal Medicine, Sahlgrenska University Hospital, SE-41345 Gothenburg, Sweden \\ ${ }^{3}$ Department of Tissue Regeneration, University of Twente, 7522 NB Enschede, The Netherlands \\ ${ }^{4}$ Department of Endocrinology and Metabolism, Leiden University Medical Center, 2300 ZA Leiden, The Netherlands \\ (Correspondence should be addressed to J Emons who is now at Department of Paediatrics, LUMC, PO Box 9600, 2300 RC Leiden, \\ The Netherlands; Email: j.emons@lumc.nl)
}

\begin{abstract}
Longitudinal bone growth is regulated in the growth plate. At the end of puberty, growth velocity diminishes and eventually ceases with the fusion of the growth plate through mechanisms that are not yet completely understood. Vascular endothelial growth factor (VEGF) has an important role in angiogenesis, but also in chondrocyte differentiation, chondrocyte survival, and the final stages of endochondral ossification. Estrogens have been shown to up-regulate VEGF expression in the uterus and bone of rats. In this study, we investigated the relation between estrogens and VEGF production in growth plate chondrocytes both in vivo and in vitro. The expression of VEGF protein was down-regulated upon ovariectomy and was restored upon
\end{abstract}

estradiol $\left(\mathrm{E}_{2}\right)$ supplementation in rat growth plates. In cultured rat chondrocyte cell line RCJ3.1C5.18, $\mathrm{E}_{2}$ dose dependently stimulated 121 and $189 \mathrm{kDa}$ isoforms of VEGF, but not the $164 \mathrm{kDa}$ isoform. Finally, VEGF expression was observed at both protein and mRNA levels in human growth plate specimens. The protein level increased during pubertal development, supporting a link between estrogens and local VEGF production in the growth plate. We conclude that estrogens regulate VEGF expression in the epiphyseal growth plate, although the precise role of VEGF in estrogen-mediated growth plate fusion remains to be clarified.

Journal of Endocrinology (2010) 205, 61-68

\section{Introduction}

Longitudinal growth occurs at the epiphyseal plate, a thin layer of cartilage entrapped between epiphyseal and metaphyseal bones, at the distal ends of the long bones (Kronenberg 2003). In the growth plate, immature cells lie toward the epiphysis, called the resting zone, with flat more mature chondrocytes in the proliferating zone and large chondrocytes in the hypertrophic zone adjacent to this. At the end of puberty, longitudinal growth ceases with total replacement of avascular cartilage by highly vascularized bone, eventually resulting in epiphyseal fusion. Estrogens are known to be important hormones in the regulation of growth plate maturation and epiphyseal fusion; they regulate and can accelerate the programmed senescence of the growth plate, leading to proliferative exhaustion of chondrocytes and epiphyseal fusion (Weise et al. 2001, Chagin \& Savendahl 2007). At a low concentration, estrogens are known to increase growth velocity, an effect possibly mediated through the GH-insulin-like growth factor 1 (IGF1) axis, while at a high concentration, estrogens inhibit growth and promote epiphyseal fusion in the long bones (Ross et al. 1986, Metzger \& Kerrigan 1994, Klein et al. 1996).

A critical step in endochondral ossification is when blood vessels enter from the primary spongiosum and osteoblasts invade from the bone marrow to lay down trabecular bone (Kember 1993, Hunziker 1994). Vascular endothelial growth factor (VEGF) is a potent mediator of angiogenesis, but it has also been shown to modulate chondrocyte differentiation and survival, osteoblast differentiation, and osteoclast recruitment (Zelzer et al. 2004, Zelzer \& Olsen 2005, Dai \& Rabie 2007). VEGF is expressed by growth plate chondrocytes and osteoblasts in different species including humans (Gerber et al. 1999, Carlevaro et al. 2000, Garcia-Ramirez et al. 2000, Haeusler et al. 2005). Human VEGF is present in six different proteins, VEGF-A, VEGF-B, VEGF-C, VEGF-D, VEGF-E, and VEGF-F, where VEGF-A has been shown to be expressed in the growth plate and also believed to be most important in the regulation of longitudinal bone growth. VEGF-A has six alternatively spliced isoforms: VEGF-121, 
VEGF-145, VEGF-165, VEGF-183, VEGF-189, and VEGF-206 (Robinson \& Stringer 2001). The receptors involved in VEGF-A signaling are VEGFR-1 (also known as fms-like tyrosine kinase receptor 1, FLT1) and VEGFR-2 (also known as kinase insert domain-containing receptor, KDR), with almost all responses being mediated through the second receptor, which has also been detected at the chondro-osseous junction in the mouse growth plate (Gerber et al. 1999, Ferrara et al. 2003, Dai \& Rabie 2007).

Inactivating VEGF in mice and monkeys resulted in impaired trabecular bone formation and expansion of the hypertrophic zone, indicating inhibition of cartilage resorption (Gerber et al. 1999, Ryan et al. 1999). In addition, Vegfa conditional knockout mice driven by a Col2a 1 promoter showed delayed invasion of blood vessels into the primary ossification center and delayed removal of terminal hypertrophic chondrocytes together with massive cell death in chondrocytes throughout the growth plate, demonstrating the importance of VEGFA in chondrocyte survival (Zelzer et al. 2004).

In vitro VEGF expression can be up-regulated by factors known to be important in the regulation of longitudinal bone growth such as fibroblast growth factor, transforming growth factor $\beta$, and IGF1 (Garcia-Ramirez et al. 2000). Studies on rats showed that in uterus and bone tissue, VEGF expression is up-regulated by estrogens (Hyder et al. 1996, Mekraldi et al. 2003). In humans, bone growth and estrogen levels increase in parallel during earlier phases of puberty, while at the end of puberty, growth ceases with a total replacement of cartilage by bone resulting in the fusion of the growth plate.

We hypothesized that estrogens have the capacity to stimulate local VEGF production in growth plate chondrocytes, and that this could be a possible mechanism involved in the process of growth plate maturation and fusion in humans. To address this, we performed in vivo studies in ovariectomized rats supplemented with estradiol $\left(\mathrm{E}_{2}\right)$ and also in vitro studies in cultured rat chondrocytes exposed to $\mathrm{E}_{2}$, and assessed chondrocyte-specific expression of VEGF. In addition, we measured VEGF expression in growth plate specimens obtained from humans in different pubertal stages.

\section{Materials and Methods}

\section{Animals and study protocol}

Female Sprague-Dawley rats were purchased from Scanbur BK AB (Sollentuna, Sweden). The animals were housed in a temperature- and humidity-controlled room under a $0600 \mathrm{~h}$ light:0600 h darkness cycle, and allowed a soy-free diet containing $0 \cdot 7 \%$ of calcium and $0.5 \%$ of phosphorus (R70; Lactamin AB, Kimstad, Sweden) and tap water ad libitum. All procedures were approved by the Ethics Committee at Göteborg University, and conformed to the National Institutes of Health Guide for the Care and Use of Laboratory Animals. The animals were randomly divided into three groups: sham operation + vehicle treatment (sham, $n=12$ ), OVX + vehicle treatment (OVX, $n=10)$, and $\mathrm{OVX}+\mathrm{E}_{2}$ treatment $\left(\mathrm{E}_{2}, n=11\right)$. At 12 weeks of age (body weight, $251 \pm 2 \mathrm{~g}$ ), the rats were either sham-operated or OVX under isoflurane anesthesia (Baxter Medical AB, Kista, Sweden), and small silastic implants were placed subcutaneously in the cervical region. The silastic implants were prepared as described previously, releasing $2.5 \mu \mathrm{g}$ /day of $\mathrm{E}_{2}$ (Vandenput et al. 2002). Vehicle-treated animals received an empty implant. $E_{2}$ was obtained from Sigma Chemical. After 6 weeks of treatment, the animals were killed by excision of the heart under isoflurane anesthesia, and the right proximal tibia was fixed in $4 \%$ paraformaldehyde, decalcified, and embedded in paraffin. Uterus size, correlating with estrogen levels, was smaller in the ovariectomized rats and slightly larger in the estrogen-supplemented animals compared with the sham-operated animals, indicating supra-physiological levels of estrogens in the estrogen-treated group. Study details were described previously (Tivesten et al. 2004, 2006).

\section{Patients and tissue preparation}

Human proximal and distal femur growth plate tissues were collected from 12 girls at different pubertal stages who were undergoing surgery for different medical indications (Table 1). One fetal sample was collected from a female donor of 23 weeks of gestational age. The study protocol was approved by the local medical ethics committees of the Leiden University Center, Leiden, The Netherlands, and by the Karolinska University Hospital, Stockholm, Sweden. Informed consent was obtained from all patients and their parents. Epiphyseal samples were either directly frozen in liquid isopentane and stored at $-80^{\circ} \mathrm{C}$ or fixed in $10 \%$ formalin, decalcified, and embedded in paraffin. All tissue samples were processed in the same way.

\section{Immunohistochemistry}

All tissues were cut into $5-\mu \mathrm{m}$ sections and mounted on histological glass slides (Starfrost, Knittel Glaser, Braunschweig, Germany), dried at $37^{\circ} \mathrm{C}$ overnight, and heated at $60^{\circ} \mathrm{C}$ for $1 \mathrm{~h}$ before immunohistochemical treatment. Immunohistochemistry was performed as described previously (Nilsson et al. 2003), with the modification that antigen retrieval was achieved by incubating with $0 \cdot 1 \%$ trypsin (Invitrogen) for $10 \mathrm{~min}$ at $37^{\circ} \mathrm{C}$. Anti-VEGF antibody was obtained from Santa Cruz Biotechnology, Inc. (Santa Cruz, CA, USA), and was used in a 1:200 dilution for rat tissues and 1:50 dilution for human tissues. Secondary anti-rabbit biotinylated antibody (Jackson ImmunoResearch Lab, West Grove, PA, USA) was used in a 1:1000 dilution, followed by incubation with avidin-biotin Vectastain $\mathrm{ABC}$ reagent according to the manufacturer's instructions (Vector Laboratories, Burlingame, CA, USA). Digital images were collected employing a Nikon Eclipse E800 microscope equipped with an Olympus DP70 digital camera. 
Table 1 Patient information and human tissues. The diagnosis of each patient, location of growth plate, age, in years, Tanner pubertal breast stage (B1-B5), and experiment in which growth plate sample was used (IHC and/or qPCR) are given in the table

\begin{tabular}{|c|c|c|c|c|}
\hline Diagnosis & Bone & Age (years) & Puberty & Experiment \\
\hline Leg length difference & Distal femur & 9 & B1-B2 & $\mathrm{IHC}$ \\
\hline Hip luxations, femur head resection & Proximal femur & 12 & $\mathrm{~B} 2$ & qPCR, IHC \\
\hline Leg length difference & Distal femur & 12 & $\mathrm{~B} 2$ & $\mathrm{IHC}$ \\
\hline Leg length difference & Distal femur & 14 & B2-B3 & $\mathrm{IHC}$ \\
\hline Hip luxations, femur head resection & Proximal femur & 13 & B3 & qPCR, IHC \\
\hline Cerebral palsy, femur head resection & Proximal femur & 15 & B4 & $\mathrm{IHC}$ \\
\hline Osteosarcoma in tibia & Distal femur & 8 & B1 & qPCR \\
\hline Upper limb amputation of the leg & Distal femur & 9 & B1 & qPCR \\
\hline Tall stature & Distal femur & 10 & $\mathrm{~B} 2$ & qPCR \\
\hline Tall stature & Distal femur & 12 & B3 & qPCR \\
\hline Tall stature & Distal femur & 15 & B4 & qPCR \\
\hline Hip luxations, femur head resection & Proximal femur & 14 & B4 & qPCR \\
\hline Fetal, selective abortion & Distal femur & 23 weeks & Fetal & qPCR \\
\hline
\end{tabular}

IHC, immunohistochemistry.

\section{Image analysis}

Images of the central two-thirds of the rat growth plates were captured in three visual fields. All pictures were taken at $200 \times$ magnification with a $2040 \times 1536$ resolution, and were further analyzed in Image Pro Plus 5.0 software (Silver Spring, MD, USA). Pictures were converted into grayscale-8 mode, and were inverted in order to obtain correct optical density (OD) values in immunopositive areas. An automatic bright object counting was performed to identify the number of immunopositive objects above the defined thresholds. Threshold level for cell size was defined as objects with an area over 12 and $20 \mu^{2}$ in the proliferative and hypertrophic zones respectively. The total OD of the immunopositive objects was calculated automatically, a function referred as density sum in the Image Pro Plus software. The analyzed areas were measured in $\mathrm{mm}^{2}$, and results are expressed as the number of positive cells $/ \mathrm{mm}^{2}$, protein expression (OD arbitrary unit) $/ \mathrm{mm}^{2}$, and protein expression (OD arbitrary unit)/per cell. Data are presented as mean \pm s.E.M.

\section{$R N A$ isolation}

Bone was removed from all epiphyseal samples, and $40-\mu \mathrm{m}$ thick sections were cut with a cryostat. Every fifth section was followed by a $5-\mu \mathrm{m}$ thick section, which was studied with hematoxylin staining to ensure lack of bone contamination. Total RNA isolation was performed with an optimized method for RNA extraction from cartilage as described by Heinrichs et al. (1994), except that the protocol was started by homogenizing the sections in $1 \mathrm{ml}$ guanidine thiocyanate solution. RNA samples from bladder and prostate tissue were obtained from Gentaur molecular products (Brussel, Belgium). RNA extraction was followed by purification using an RNeasy kit according to the manufacturer's protocol (Qiagen), and the quality and integrity of each sample were checked with the Agilent 2100 Bioanalyzer.

\section{Real-time reverse transcription-PCR}

RNA was reverse transcribed into cDNA using a First Strand cDNA Synthesis kit for quantitative PCR (qPCR; Roche Diagnostics Gmbh) according to the manufacturer's instructions. Expression of $V E G F-A$ and $V E G F R-2(K D R)$ mRNA was quantified by real-time PCR using the Bio-Rad iCycler with SYBR Green. QuantiTect Primer Assays were purchased from Qiagen Benelux BV, and were used according to the manufacturer's protocol. Threshold cycles $\left(C_{t}\right)$ were estimated and averaged for the triplicates. Relative amounts of mRNA were normalized to $\beta_{2}$-microglobulin expression in the same sample to account for variability in the initial concentration and quality of total RNA and in the efficiency of the reverse transcription reaction.

\section{Western blotting}

Nontransformed clonal rat chondrogenic cells RCJ3.1C5.18 (C5.18 cells) were differentiated for 10 days (Lunstrum et al. 1999), and were subsequently treated for $24 \mathrm{~h}$ with a dose range of $E_{2}$. Cells were lysated, and the protein concentration was measured by the Bradford protein assay (Bio-Rad Laboratories $\mathrm{AB}$ ). Proteins were separated on acrylamide gels (Bio-Rad Laboratories), and transferred to polyvinylidene fluoride membrane. Three different isoforms of VEGFA were detected employing anti-VEGF rabbit Ab (1:1500; sc-152, Santa Cruz Biotechnology Inc). Secondary goat antirabbit antibody was peroxidase labeled and used in a 1:10 000 dilution (Santa Cruz Biotechnology Inc). The resulting bands 
were confirmed by comparing the size of the protein in the cell extract with that of the known molecular weight markers. The antigen-antibody complexes were then detected by chemiluminescence. After the films had been developed, blots were stained with Coomassie blue to ensure equal loading of total protein. Density measurements were normalized per density of Coomassie blue staining (Chrysis et al. 2005). Each experiment was repeated at least three times.

\section{Statistical analysis}

The human sections were blinded, and a relative staining intensity was scored (score 0-3) for the proliferative and hypertrophic zones of each growth plate. Scores were displayed in a scatter plot, and a linear regression analysis was performed to calculate significance. The same was done for the qPCR results.

For the rat data, six growth plate sections for every animal were analyzed by the image analysis protocol described above, and means were calculated in terms of VEGF-positive cells $/ \mu \mathrm{m}^{2}$
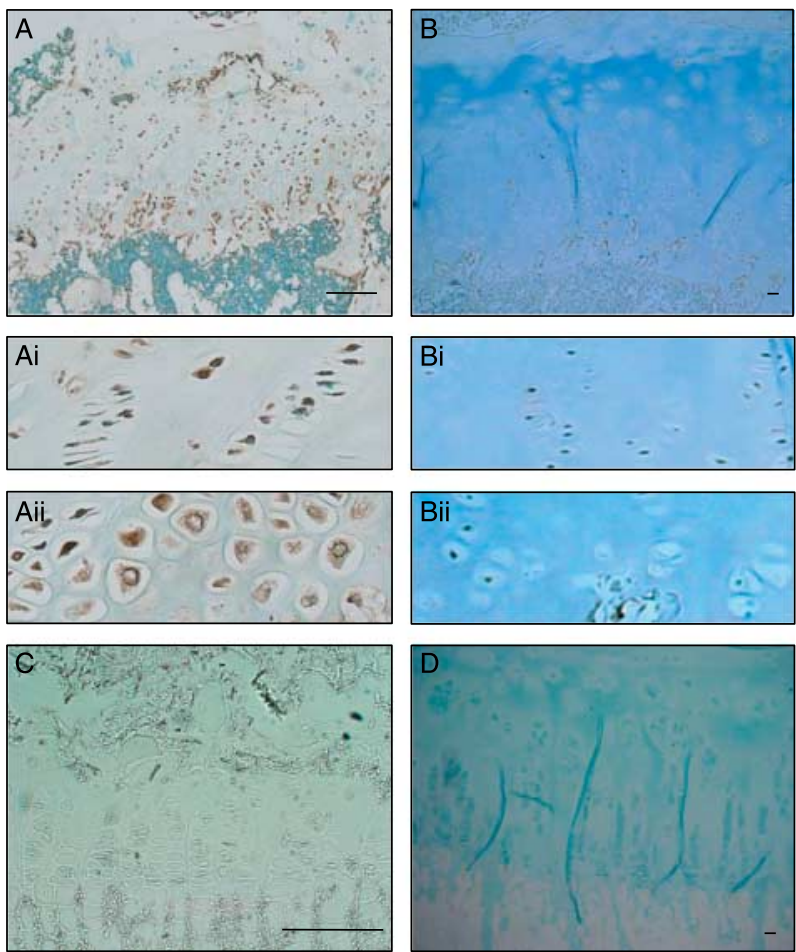

Figure 1 VEGF immunohistochemistry staining. VEGF protein expression was detected in the rat growth plate (panel $A$, $100 \times$ magnification) and the human pubertal growth plate (panel $B, 100 \times$ magnification). Panels $A i$ and $B i$ show $5 \times$ enlargements of the proliferative zone in panels $\mathrm{A}$ and $\mathrm{B}$. Panels Aii and Bii show $5 \times$ enlargements of the hypertrophic zone in panels $A$ and $B$. Pre-incubation of the primary antibody with recombinant VEGF abolished the staining in both proliferative and hypertrophic chondrocytes (panel C, $400 \times$ ). Panel D shows a negative control for the human growth plates. Bars indicate $100 \mu \mathrm{m}$.
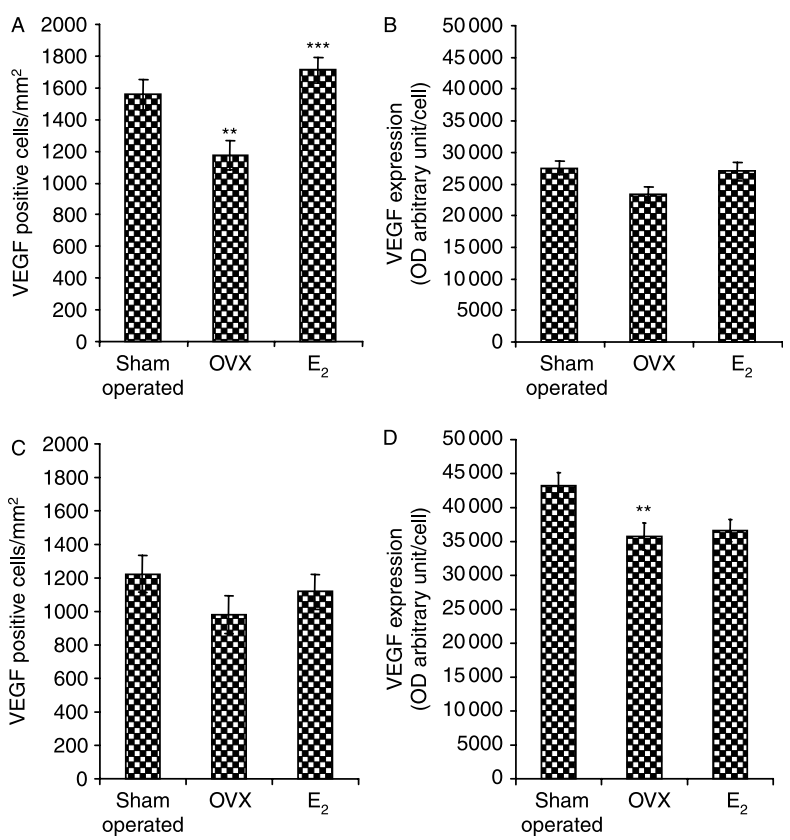

Figure 2 Quantification of VEGF staining in the proliferative and hypertropic zones. VEGF staining was quantified with a computerized method for the proliferative zone. Panel A shows a significant decrease in the amount of positive cells $/ \mathrm{mm}^{2}$ growth plate after ovariectomy, which was completely restored by $17 \beta-$ estradiol. Staining intensity per cell (OD arbitrary unit/cell) showed no difference between all groups (panel B). The hypertrophic zone showed the same trend as the proliferative zone; however, there were no statistically significant differences (panel C). Staining intensity per cell (OD arbitrary unit/cell) showed a significant decrease after ovariectomy in the hypertropic zone, but there was no restoration with estrogen supplementation (panel D). Data are presented as mean \pm s.E.M. ${ }^{*} P<0 \cdot 05,{ }^{* *} P<0 \cdot 01$, and ${ }^{* * *} P<0 \cdot 001$.

and VEGF expression (OD arbitrary unit)/per cell for each animal. Significance was calculated by one-way ANOVA followed by Fisher's protected least significant difference test.

\section{Results}

\section{$V E G F$ protein expression in the rat growth plate}

Sham-operated rats were used as an internal control which confirmed abundant VEGF expression in the growth plates, in both the proliferative and hypertrophic zones (Fig. 1A). Pre-incubation of the primary antibody with recombinant VEGF abolished the staining in both proliferative and hypertrophic chondrocytes (Fig. 1C). Staining was analyzed by a computerized method for the proliferative and hypertrophic zones (see Materials and Methods section).

To reveal any possible regulation of VEGF expression by estrogens, we analyzed the number of VEGF-expressing chondrocytes in rats upon ovariectomy and $\mathrm{E}_{2}$ supplementation. Ovariectomy resulted in a significant decrease in the number of VEGF-positive cells in the proliferative zone 

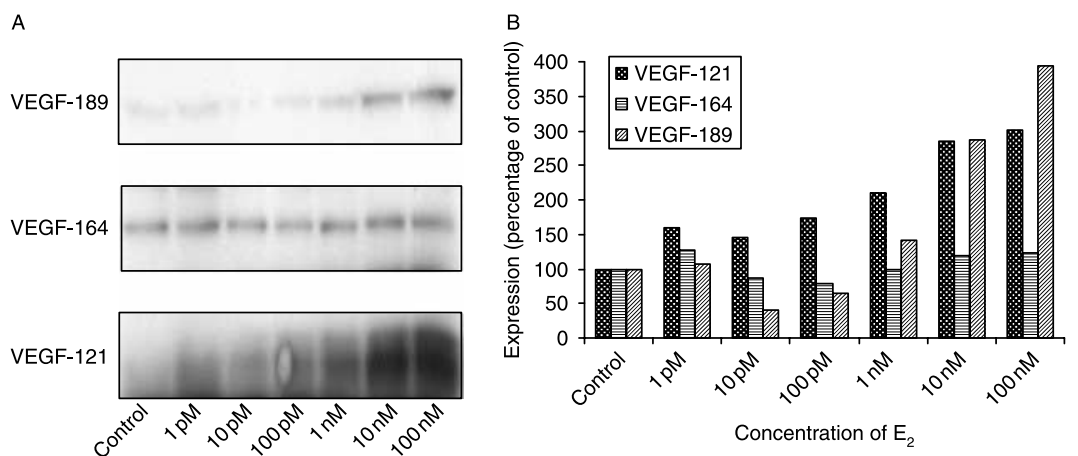

Figure 3 VEGFA isoform western blot analysis in chondrocyte culture. Panel A illustrates that $17 \beta$-estradiol directly stimulated the expression of isoforms VEGF-189, VEGF-164, and VEGF-121. The isoforms VEGF-121 and VEGF-189 showed a very strong response, and in contrast, VEGF-164 showed just a marginal response. In panel B, the expression levels are calculated as a percentage of the VEGF expression in the nontreated control.

$\left(1173 \pm 93\right.$ vs $1556 \pm 100$ cells $/ \mathrm{mm}^{2}$ in sham-operated animals; $P<0 \cdot 01)$, an effect that was completely restored by $E_{2}$ replacement $\left(1713 \pm 81\right.$ vs $1173 \pm 93$ cells $/ \mathrm{mm}^{2}$ in vehicle alone; $P<0 \cdot 001 ;$ Fig. $2 \mathrm{~A})$. A similar trend was observed in hypertropic chondrocytes, albeit not statistically significant (Fig. 2C). The level of VEGF per cell (expressed as OD arbitrary units/cell) did not differ significantly between the groups in the proliferative zone (Fig. 2B). However, in the hypertropic zone, ovariectomy resulted in a significant decrease in the level of VEGF per cell $(35747 \pm 1989$ vs $43240 \pm 1900$ in sham-operated animals; $P<0 \cdot 01)$, an effect which was not restored by $\mathrm{E}_{2}$ supplementation (36 601 \pm 1615 vs $35747 \pm 1989$ in vehicle alone; $P=0 \cdot 74$; Fig. 2D).

\section{VEGF-A isoform expression in cultured chondrocytes}

In order to distinguish between direct and systemic effects of estrogens on VEGF expression, we performed experiments in the rat chondrogenic cell line RCJ3.1C5.18 (C5.18 cells), which can be differentiated into hypertrophic chondrocytes (Lunstrum et al. 1999). The cells were differentiated for 10 days and were then treated with $\mathrm{E}_{2}$ for $24 \mathrm{~h} . \mathrm{E}_{2}$ dose dependently stimulated the expression of VEGF-121 and VEGF-189, while VEGF-164 expression was not affected (Fig. 3). VEGF-189 was suppressed by low $\mathrm{E}_{2}$ concentrations, and it was increased by high concentrations.

\section{$V E G F$ protein and $m R N A$ expressions in human growth plates}

Growth plate biopsies were obtained from girls at different stages of pubertal development. To verify how VEGF is distributed in the human pubertal growth plate, we analyzed VEGF expression levels in these rare tissue samples. Of the 13 collected growth plates, we analyzed six human growth plates for VEGF protein expression and nine for VEGF mRNA expression (Table 1). In all these human growth plates, VEGF protein was detected in both proliferative and hypertrophic zone chondrocytes (Fig. 1B). When the relative staining intensity was scored (score 0-3), a significant increase in VEGF expression with progression of puberty was found in both the proliferative $(P=0.022)$ and hypertrophic zones $(P=0 \cdot 017$; Fig. 4 panels A and B). Negative controls showed no staining (data not shown).

Studies of mRNA levels with qPCR confirmed VEGF expression in pubertal as well as fetal human growth plates, albeit expression of $V E G F \mathrm{mRNA}$ in the prepubertal growth plate was $\sim 200$-fold lower compared with the expression in prostate and bladder tissues (positive controls). The $C_{\mathrm{t}}$ for VEGF expression were subtracted from the $\beta 2$-microglobulin $C_{\mathrm{t}}$ in order to calculate the $\Delta C_{\mathrm{t}}$. Average values for the different groups were calculated and compared with the prepubertal growth plate. The fetal growth plate showed a 4-2-fold higher expression of VEGF compared with the prepubertal growth plate. The pubertal growth plate samples $(n=6)$ showed on average a $1 \cdot 6$-fold higher expression of VEGF mRNA compared with the prepubertal growth plate $(n=2)$, but in contrast to VEGF protein expression, we did not find a significant correlation between VEGF mRNA levels assessed by qPCR and the stage of pubertal development $(P=0 \cdot 183, R=0 \cdot 238)$. The VEGF receptor,
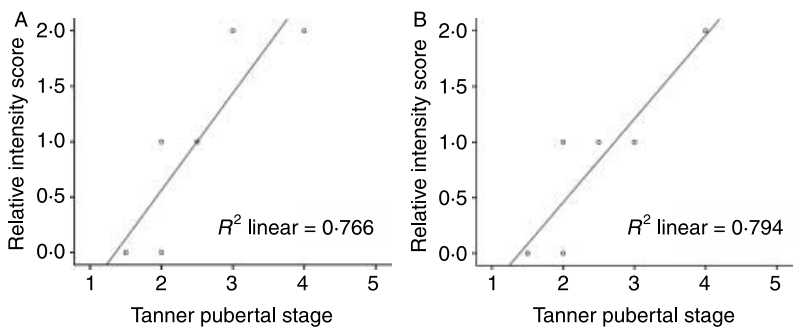

Figure 4 Scatter plot of VEGF expression in the human growth plate. Relative intensity of VEGF protein expression was scored for each growth plate and plotted in relation to the pubertal stage for the proliferative zone (panel A) and the hypertrophic zone (panel B). $R^{2}$ values were respectively $0 \cdot 766(P=0 \cdot 022)$ for the proliferative zone and $0.794(P=0 \cdot 017)$ for the hypertrophic zone. 
VEGFR2, was also expressed at mRNA level in all our growth plate samples that were analyzed. Similar to VEGF, the average VEGFR2 mRNA level was 1.6-fold higher in pubertal girls $(n=6)$ compared with the prepubertal girls $(n=2)$, but there was no statistically significant correlation with pubertal progression $(P=0 \cdot 585, R=0 \cdot 045)$ likely due to a high variation between samples and low number of patients.

\section{Discussion}

Our in vivo and in vitro data demonstrate that $\mathrm{E}_{2}$ directly stimulates the expression of VEGF in rat growth plate chondrocytes. Furthermore, we confirmed that VEGF is expressed in the human pubertal growth plate and that the VEGF protein level increases with pubertal progression, supporting a link between estrogens and local VEGF production in the growth plate.

VEGF was previously detected mostly in hypertrophic chondrocytes of human growth plate samples by immunohistochemistry (Haeusler et al. 2005). We observed VEGF expression not only in the hypertropic zone, but also in the proliferative zone, which is in line with the observations of Horner et al. who studied VEGF protein expression in neonatal human growth plate cartilage (Horner et al. 1999). VEGF mRNA was also detected in the proliferative zone of murine growth plate cartilage (Cramer et al. 2004). VEGF expression in proliferating chondrocytes and the significant change in expression with alternating estrogen levels were observed in both human and rat growth plates in our study. This slight divergence in results could be due to technical issues attributed to immunohistochemistry such as antigen retrieval or type of antibody.

We confirmed our immunohistochemistry data by qPCR analysis. mRNA expression of VEGF and the VEGFR-2 was, to our knowledge for the first time, detected in adolescent and pubertal human growth plates. VEGF mRNA was previously detected in the fetal growth plate by others (Garcia-Ramirez et al. 2000, Petersen et al. 2002). Expression of VEGFR-2 was detected earlier at the chondroosseous junction in mice (Gerber et al. 1999), in epiphyseal cartilage of pigs (Kim et al. 2009), in the avian growth plate (Rath et al. 2007), and in hypertrophic chondrocytes of the fetal growth plate (Petersen et al. 2002). In contrast to protein levels, mRNA did not reveal a significant increase in VEGF expression with progression of puberty. This discrepancy in results could be due to a difference between mRNA and protein expression, a difference in tissue preparation for RNA extraction when the surrounding bone was removed or alternatively due to a change in morphological organization of the growth plate during progression of puberty (e.g. a decreased hypertrophic layer).

This is the first report to demonstrate a link between estrogens and VEGF expression in the epiphyseal growth plate. In vivo treatment of rats with $\mathrm{E}_{2}$ increased the number of growth plate chondrocytes expressing VEGF. In line with this, in the hypertrophic zone of ovariectomized animals, the number of VEGF-positive cells $/ \mathrm{mm}^{2}$ growth plate was decreased. In addition, in vitro data in a rat chondrocytic cell line showed a dose-dependent stimulatory effect of $E_{2}$ on the expression of VEGF-121 isoform. VEGF-189 was suppressed by low $\mathrm{E}_{2}$ concentrations and was stimulated by higher concentrations. This might counterbalance the slight concomitant increase of VEGF-121, thereby protecting the growth plate when exposed to low concentrations of $\mathrm{E}_{2}$. Our growth plate findings are in line with previous reported effects of estrogens on VEGF expression in bone, uterus, and breast cancer tissues (Mekraldi et al. 2003, Kazi et al. 2005, Garvin et al. 2006).

Systemic estrogen levels increase with puberty eventually resulting in epiphyseal fusion by the end of puberty (Juul 2001), presumably due to acceleration of growth plate senescence through proliferative exhaustion of chondrocytes (Weise et al. 2001). From our results obtained in rats, we hypothesized that estrogens not only accelerate senescence of the growth plate, but also stimulate chondrocytes to secrete VEGF, which might contribute to the process of epiphyseal fusion. VEGF protein was detected in human pubertal growth plates, and indeed, the expression level significantly increased during pubertal progression. This observation in humans supports our findings in rats, and strengthens our hypothesis that estrogens stimulate VEGF expression in the growth plate.

A 200-fold lower VEGF mRNA level in the growth plate was observed compared with the prostate or bladder tissues. We believe that the observed stimulation of VEGF expression by estrogens in avascular growth plate chondrocytes can substantially affect the growth plate. Indeed, VEGF has an important role in chondrocyte differentiation, chondrocyte survival, and endochondral ossification (Zelzer et al. 2004, Zelzer \& Olsen 2005, Dai \& Rabie 2007). In several studies, inhibition of VEGF showed dramatic effects on the growth plate, such as expansion of the hypertrophic zone and delayed removal of terminal hypertrophic chondrocytes (Gerber et al. 1999, Zelzer et al. 2004). Conversely, one might speculate that an increase in expression leads to a smaller hypertrophic zone, a more rapid removal of terminal hypertrophic chondrocytes, and eventually epiphyseal fusion. To our knowledge, reports on an increase in VEGF expression have not been published before.

Estrogen levels were higher in the estrogen-supplemented rats compared with the sham-operated rats. Uterus size, correlating with estrogen levels, was small in ovariectomized rats compared with the sham-operated animals and was slightly larger in the estrogen-supplemented animals, indicating supra-physiological levels of estrogens in the estrogen-treated group (Tivesten et al. 2004, 2006). Serum levels of $E_{2}$ were not measured in patients from whom growth plate tissue samples were collected. However, serum levels of $E_{2}$ are well known to positively correlate with the stage of pubertal development (Norjavaara et al. 1996). 
The collection of human samples is small and originates from patients having a variability of disorders. However, human growth plate samples are extremely difficult to obtain. We believe that even though patients suffered from diverse disorders, the underlying mechanism of epiphyseal maturation and fusion will be the same for all growth plates. Eventually, longitudinal growth stops in all patients, with only few exceptions, at the end of puberty. The human data are in line with both in vivo and in vitro rat data, thereby strengthening our conclusion that estrogens stimulate VEGF expression in the growth plate. Estrogen levels were not analyzed in these patients, and our assumption of different levels of estrogen exposure is based on the fact that tissue samples were obtained from girls in different pubertal stages.

In summary, we demonstrated that VEGF protein expression in the growth plate is elevated by estrogens in vivo in ovariectomized rats and in vitro in a rat chondrocytic cell lines. Our findings are supported by human expression studies in girls in different pubertal stages. From this, we conclude that estrogens stimulate VEGF expression in the growth plate, although the exact role of VEGF in estrogenmediated growth plate fusion remains to be clarified.

\section{Declaration of interest}

The authors declare that there is no conflict of interest that could be perceived as prejudicing the impartiality of the research reported.

\section{Funding}

This study was supported by ZonMw (project \# 920-03-358), The Netherlands; the Swedish Research Council (project 2007-54X15073-04-3); and a visiting scholarship award from the European Society for Paediatric Endocrinology.

\section{Acknowledgements}

The authors thank the orthopedic surgeons in the Leiden University Medical Center and at the Karolinska University Hospital in Stockholm for providing the growth plate samples.

\section{References}

Carlevaro MF, Cermelli S, Cancedda R \& Descalzi CF 2000 Vascular endothelial growth factor (VEGF) in cartilage neovascularization and chondrocyte differentiation: auto-paracrine role during endochondral bone formation. Journal of Cell Science 113 59-69.

Chagin AS \& Savendahl L 2007 Estrogens and growth: review. Pediatric Endocrinology Reviews 4 329-334.

Chrysis D, Zaman F, Chagin AS, Takigawa M \& Savendahl L 2005 Dexamethasone induces apoptosis in proliferative chondrocytes through activation of caspases and suppression of the Akt-phosphatidylinositol 3'-kinase signaling pathway. Endocrinology 146 1391-1397.

Cramer T, Schipani E, Johnson RS, Swoboda B \& Pfander D 2004 Expression of VEGF isoforms by epiphyseal chondrocytes during low-oxygen tension is HIF-1 $\alpha$ dependent. Osteoarthritis Cartilage 12 433-439.

Dai J \& Rabie AB 2007 VEGF: an essential mediator of both angiogenesis and endochondral ossification. Journal of Dental Research 86 937-950.

Ferrara N, Gerber HP \& LeCouter J 2003 The biology of VEGF and its receptors. Nature Medicine 9 669-676.
Garcia-Ramirez M, Toran N, Andaluz P, Carrascosa A \& Audi L 2000 Vascular endothelial growth factor is expressed in human fetal growth cartilage. Journal of Bone and Mineral Research 15 534-540.

Garvin S, Nilsson UW, Huss FR, Kratz G \& Dabrosin C 2006 Estradiol increases VEGF in human breast studied by whole-tissue culture. Cell Tissue Research 325 245-251.

Gerber HP, Vu TH, Ryan AM, Kowalski J, Werb Z \& Ferrara N 1999 VEGF couples hypertrophic cartilage remodeling, ossification and angiogenesis during endochondral bone formation. Nature Medicine 5 623-628.

Haeusler G, Walter I, Helmreich M \& Egerbacher M 2005 Localization of matrix metalloproteinases, (MMPs) their tissue inhibitors, and vascular endothelial growth factor (VEGF) in growth plates of children and adolescents indicates a role for MMPs in human postnatal growth and skeletal maturation. Calcified Tissue International 76 326-335.

Heinrichs C, Yanovski JA, Roth AH, Yu YM, Domene HM, Yano K, Cutler GB Jr \& Baron J 1994 Dexamethasone increases growth hormone receptor messenger ribonucleic acid levels in liver and growth plate. Endocrinology 135 1113-1118.

Horner A, Bishop NJ, Bord S, Beeton C, Kelsall AW, Coleman N \& Compston JE 1999 Immunolocalisation of vascular endothelial growth factor (VEGF) in human neonatal growth plate cartilage. Journal of Anatomy $194519-524$.

Hunziker EB 1994 Mechanism of longitudinal bone growth and its regulation by growth plate chondrocytes. Microscopy Research and Technique $\mathbf{2 8}$ 505-519.

Hyder SM, Stancel GM, Chiappetta C, Murthy L, Boettger-Tong HL \& Makela S 1996 Uterine expression of vascular endothelial growth factor is increased by estradiol and tamoxifen. Cancer Research 56 3954-3960.

Juul A 2001 The effects of oestrogens on linear bone growth. Human Reproduction Update 7 303-313.

Kazi AA, Jones JM \& Koos RD 2005 Chromatin immunoprecipitation analysis of gene expression in the rat uterus in vivo: estrogen-induced recruitment of both estrogen receptor alpha and hypoxia-inducible factor 1 to the vascular endothelial growth factor promoter. Molecular Endocrinology 19 2006-2019.

Kember NF 1993 Cell kinetics and the control of bone growth. Acta Paediatrica 82 (Suppl 391) 61-65.

Kim HK, Bian H, Aya-ay J, Garces A, Morgan EF \& Gilbert SR 2009 Hypoxia and HIF-1alpha expression in the epiphyseal cartilage following ischemic injury to the immature femoral head. Bone 45 280-288.

Klein KO, Martha PM Jr, Blizzard RM, Herbst T \& Rogol AD 1996 A longitudinal assessment of hormonal and physical alterations during normal puberty in boys. II. Estrogen levels as determined by an ultrasensitive bioassay. Journal of Clinical Endocrinology and Metabolism 81 3203-3207.

Kronenberg HM 2003 Developmental regulation of the growth plate. Nature 423 332-336.

Lunstrum GP, Keene DR, Weksler NB, Cho YJ, Cornwall M \& Horton WA 1999 Chondrocyte differentiation in a rat mesenchymal cell line. Journal of Histochemistry and Cytochemistry 47 1-6.

Mekraldi S, Lafage-Proust MH, Bloomfield S, Alexandre C \& Vico L 2003 Changes in vasoactive factors associated with altered vessel morphology in the tibial metaphysis during ovariectomy-induced bone loss in rats. Bone $\mathbf{3 2}$ 630-641.

Metzger DL \& Kerrigan JR 1994 Estrogen receptor blockade with tamoxifen diminishes growth hormone secretion in boys: evidence for a stimulatory role of endogenous estrogens during male adolescence. Journal of Clinical Endocrinology and Metabolism 79 513-518.

Nilsson O, Chrysis D, Pajulo O, Boman A, Holst M, Rubinstein J, Martin RE \& Savendahl L 2003 Localization of estrogen receptors-alpha and -beta and androgen receptor in the human growth plate at different pubertal stages. Journal of Endocrinology 177 319-326.

Norjavaara E, Ankarberg C \& Albertsson-Wikland K 1996 Diurnal rhythm of 17 beta-estradiol secretion throughout pubertal development in healthy girls: evaluation by a sensitive radioimmunoassay. Journal of Clinical Endocrinology and Metabolism 81 4095-4102.

Petersen W, Tsokos M \& Pufe T 2002 Expression of VEGF121 and VEGF165 in hypertrophic chondrocytes of the human growth plate and epiphyseal cartilage. Journal of Anatomy 201 153-157. 
Rath NC, Huff WE \& Huff GR 2007 Thiram-induced changes in the expression of genes relating to vascularization and tibial dyschondroplasia. Poultry Science 86 2390-2395.

Robinson CJ \& Stringer SE 2001 The splice variants of vascular endothelial growth factor (VEGF) and their receptors. Journal of Cell Science 114 853-865.

Ross JL, Long LM, Skerda M, Cassorla F, Kurtz D, Loriaux DL \& Cutler GB Jr 1986 Effect of low doses of estradiol on 6-month growth rates and predicted height in patients with Turner syndrome. Journal of Pediatrics 109 950-953.

Ryan AM, Eppler DB, Hagler KE, Bruner RH, Thomford PJ, Hall RL, Shopp GM \& O'Neill CA 1999 Preclinical safety evaluation of rhuMAbVEGF, an antiangiogenic humanized monoclonal antibody. Toxicologic Pathology 27 78-86.

Tivesten A, Moverare-Skrtic S, Chagin A, Venken K, Salmon P, Vanderschueren D, Savendahl L, Holmang A \& Ohlsson C 2004 Additive protective effects of estrogen and androgen treatment on trabecular bone in ovariectomized rats. Journal of Bone and Mineral Research 19 1833-1839.

Tivesten A, Bollano E, Nystrom HC, Alexanderson C, Bergstrom G \& Holmang A 2006 Cardiac concentric remodelling induced by non-aromatizable (dihydro-)testosterone is antagonized by oestradiol in ovariectomized rats. Journal of Endocrinology 189 485-491.
Vandenput L, Boonen S, Van Herck E, Swinnen JV, Bouillon R \& Vanderschueren D 2002 Evidence from the aged orchidectomized male rat model that 17 beta-estradiol is a more effective bone-sparing and anabolic agent than 5alpha-dihydrotestosterone. Journal of Bone and Mineral Research 17 2080-2086.

Weise M, De Levi S, Barnes KM, Gafni RI, Abad V \& Baron J 2001 Effects of estrogen on growth plate senescence and epiphyseal fusion. PNAS $\mathbf{9 8}$ 6871-6876.

Zelzer E \& Olsen BR 2005 Multiple roles of vascular endothelial growth factor (VEGF) in skeletal development, growth, and repair. Current Topics in Development Biology 65 169-187.

Zelzer E, Mamluk R, Ferrara N, Johnson RS, Schipani E \& Olsen BR 2004 VEGFA is necessary for chondrocyte survival during bone development. Development 131 2161-2171.

Received in final form 28 December 2009

Accepted 19 January 2010

Made available online as an Accepted Preprint 19 January 2010 